\title{
Die Bedeutung der Hochertragssorten im Entwicklungsprozeß der indischen Landwirtschaft
}

\section{Der Zwang zur anbaumäßigen Intensivierung}

In den letzten vier Jahrzehnten hat sich die Bevölkerung Indiens mehr als verdoppelt. Die Zuwachsraten stiegen kontinuierlich an: 6\% von 1901 bis $1911,14 \%$ von 1941 bis $1951,22 \%$ von 1951 bis $1961,25 \%$ von 1961 bis 1971. Indiens Einwohnerzahl von gegenwärtig 600 Millionen Menschen umfaßt mehr als 15\% der Erdbevölkerung, die auf etwas über $2 \%$ der Landoberfläche der Erde dicht zusammengedrängt wohnen.

Den Wirtschaftsplanern ist es nicht gelungen, die jährlich benötigten Millionen von Arbeitsplätzen bereitzustellen, die nötig gewesen wären, um das gewaltige Heer der Arbeitslosen nicht weiter anwachsen zu lassen.

Der Anteil der in der Landwirtschaft Beschäftigten hat sich seit der Unabhängigkeit 1947 praktisch nicht verändert und liegt bei $80 \%$. In Indien, wie in den meisten Ländern der Dritten Welt, nimmt nicht die Industrie, sondern die Landwirtschaft die dominierende wirtschaftliche Stellung ein. Es ist aber nicht so, daß zwischen der Entwicklung der Industrie und der Landwirtschaft eine grundsätzliche theoretische Antinomie herrscht. Beide sind in Wirklichkeit nur die Seiten einer Medaille, nämlich des einen fundamentalen Problems: der Modernisierung rückständiger, nicht leistungsfähiger wirtschaftlicher und sozialer Institutionen. Die Probleme, die mit der Modernisierung der Landwirtschaft zusammenhängen, sind in der Regel allerdings komplexer und schwieriger zu bewältigen als jene des Industriesektors. Mit dem normativen Terminus der Modernisierung sollen im folgenden positive Veränderungen in den sozialökonomischen Verhältnissen der Mehrheit der Bevölkerung verstanden werden. Darunter sind elementare Grundbedürfnisse wie Nahrung, Wohnen, Gesundheit und Ausbildung zu verstehen, aber auch das Recht auf Arbeit, eine minimale soziale Sicherheit und Chancengleichheit.

Veränderungen sollen darum alle Lebensbereiche eines Landes umfassen, weil sonst lediglich neue wirtschaftliche und schließlich politische Ungleichgewichte und Dualismen geschaffen werden, die leicht einen Rückfall in eine erneute Stagnation von Wirtschaft und Gesellschaft verursachen können (1).

1973/74 betrug der Anteil von Land- und Forstwirtschaft am Volkseinkommen $46 \%$, gegenüber $14,5 \%$ von Industrie und Bergbau (2). Da sich die Verhält- nisse in absehbarer Zeit nicht entscheidend ändern werden, bedeutet dies, daß der Entwicklung der Landwirtschaft auf lange Sicht für die Lösung wirtschaftlicher Probleme Indiens erste Priorität einzuräumen ist (3).

$\mathrm{Da}$ im Falle Indiens zusätzlich bebaubare Flächen in Form von Neu- und Brachland nur in begrenztem Ausmaße zur Verfügung stehen oder nur mit großem Kapitalaufwand nutzbar gemacht werden können (4), besteht bei heutiger Überbevölkerung auf dem Lande ein sich rasch verschärfender Zwang zur Steigerung der Flächenproduktivität und somit zur Intensivierung der indischen Landwirtschaft.

Während sich die Bevölkerung von 1951 bis 1974 um $61 \%$ erhöhte, stieg die mit Nahrungsmitteln bebaute Fläche in der selben Zeit um $27 \%$. Als Folge davon weist die bewirtschaftete Fläche pro Kopf der landwirtschaftlichen Bevölkerung während den letzten 20 Jahren eine abnehmende Tendenz auf. Selbst wenn alle denkbaren Landreserven genutzt werden könnten, würde im Jahre 2000 bei einer prognostizierten Bevölkerung von 950 Millionen das bewirtschaftete Land pro Kopf von heute 0,25 Hektaren auf 0,2 Hektaren sinken.

Da sich die Zahl der landwirtschaftlichen Betriebe nicht verringerte, ist auch die durchschnittliche Betriebsgröße erwartungsgemäß gesunken und die Anbauflächen nach Produkten sind bis auf wenige Ausnahmen konstant geblieben (5).

\section{Drei Fragen zur Bedeutung neuer Getreide- sorten}

Die Bauernfamilie eines Dorfes im agrarisch nicht begünstigten indischen Bundesstaat Orissa, hat sich auf dem folgenden Farbbild (6) offenbar dazu entschlossen, auf einem seiner regenbewässerten Grundstücke einen Brunnen zu graben. Durch gesicherte Wasserzufuhr soll in Zukunft eine agrarische Ertragsverbesserung erzielt werden. Statt einer einzigen Reis- oder Weizenernte pro Jahr sollen zwei oder gar drei möglich werden. Der Bauer geht ein großes Risiko ein: wird er überhaupt Wasser finden? In Indien bleiben die Hälfte der von Hand erstellten Brunnen trocken. Der Arbeitsaufwand und damit die Kosten eines

Walter Rambousek, dipl. nat., Assistent, Geographisches Institut der Universität Zürich, Blümlisalpstr. 10, 8006 Zürich 
Brunnens schwanken je nach Bodenbeschaffenheit und Tiefe des Grundwasserspiegels ganz beträchtlich. Die Zinsen- und Schuldenlasten sind selbst bei staatlichen Darlehen enorm hoch und können nur dann reduziert werden, wenn von Anfang an erfolgreich für den Markt produziert wird. Erfolgreich produzieren heißt in unserem Zusammenhang, die günstigsten Saatsorten zu verwenden, den richtigen Dünger angemessen und rechtzeitig zu erhalten und richtig der Erde beizumessen, fachgerecht $\mathrm{zu}$ bewässern und schließlich durch sorgfältiges Ernten und Lagern die Ertragsverluste zu minimieren.

Landwirtschaftliche Berater werden vor allem bei jenen Bauern von entscheidender Bedeutung, die als erste versuchen, neue Anbaupraktiken einzuführen. Neben den eigenen Familienangehörigen helfen Freunde aus dem Dorfe bei der Arbeit mit. Da die Grabungsarbeiten in der Regel während der arbeitsarmen Trockenzeit, meist zwischen Januar und Mai, ausgeführt werden, sind auch Landarbeiter in genügender Zahl und zu gedrückten Tageslöhnen verfügbar. Das Aushubmaterial wird von Frauen in Körben auf dem Kopf weggetragen, was später, wenn felsiges Material ausgebrochen wird und die Temperaturen im April und Mai auf über $40 \mathrm{Grad}$ ansteigen, eine anstrengende Arbeit ist.

Für eine leistungsfähige Bewässerung allerdings bedarf der Bauer einer Diesel- oder Elektropumpe. Wie drei Viertel der 600000 Dörfer Indiens verfügt auch die im Hintergrund erkennbare Siedlung über keinen elektrischen Strom. Die Versorgung mit Diesel ist unregelmäßig und viel zu teuer. Es bleibt die Handbewässerung.

Getreidehalme liegen aufgetürmt vor dem Haus. Sie dienen als Futtermittel für das Vieh und haben bis zur nächsten Ernte auszureichen, falls die erfolgreiche Brunnenbewässerung nicht früher einen zusätzlichen Ertrag liefert. Die meisten Hochertragssorten zeichnen sich unter anderem dadurch aus, daß sie wesentlich kürzere Halme als die traditionellen Getreidearten aufweisen und der Bauer dadurch quantitativ weniger Futtermittel erhält. Für den Innovator gilt es abzuwägen, ob die Mehrerträge mindestens den dadurch bedingten Einkauf an Futtermitteln aufwiegen. Nicht der indische Bauer ist a priori rückständig und seine Risikofreude beschränkt, sondern die Entscheidungen, denen er gegenübersteht, wollen vorsichtig getroffen sein.
Es geht im folgenden nicht darum, die angedeuteten Schwierigkeiten bei der Einführung einer einzigen grundlegenden Voraussetzung für eine Modernisierung der indischen Landwirtschaft abzuhandeln. Da die Entwicklung der indischen Landwirtschaft, wie wir früher feststellten, primär von der Intensivierung der heutigen Nutzung abhängt, möchten wir im definierten Sinne eines agrarischen Modernisierungsprozesses, der einer möglichst breiten Schicht von Bauern zu gute kommt, vielmehr anzudeuten versuchen, wie weit sich erstens die Einführung von Hochertragssorten bei den Nahrungsmittelgetreiden hat verbreiten können. Wir fragen, wievielen Bauern es offensichtlich gelungen ist, die innovatorischen Hemmnisse zu überwinden, die wir im einzelnen an dieser Stelle nicht untersuchen (7).

Zweitens interessiert, welche Gruppen von Bauern davon am meisten haben profitieren können und drittens schließlich soll im Sinne einer Zusammenfassung die bisherige Bedeutung der Hochertragssorten im landwirtschaftlichen Entwicklungsproze $\beta$ gewichtet und die Antwort versucht werden, ob von der Strategie der «Grünen Revolution» in Zukunft entscheidende modernistische Innovationen $\mathrm{zu}$ erwarten sind (8).

\section{Verbreitung und Erfolge der Hochertragssorten}

Wenn wir die rasch anwachsende landwirtschaftliche Produktion zwischen 1967/68 und 1970/71 betrachten, erstaunt es nicht, daß die ersten Jahre der Grünen Revolution zu Optimismus berechtigten. Während die Produktion an Nahrungsmittelgetreide von 88,9 Millionen Tonnen 1964/65 auf 95 Millionen Tonnen 1967/68 stieg und 1970/71 gar mit 108,4 Millionen Tonnen angegeben wurde, scheint der Begriff «Revolution» vorerst angebracht (9).

Eine Möglichkeit, die Bedeutung der mengenmäßigen Zuwachsraten seit 1967/68 zu beurteilen, bietet sich im Vergleich von langfristigen Wachstumsraten: von $1949 / 50$ bis $1964 / 65$ betrug die Zunahme der Nahrungsmittelproduktion im jährlichen Durchschnitt $3,05 \%$, während sich jene von $1949 / 50$ bis $1970 / 71$ unter Ausschluß der beiden katastrophalen Dürrejahre 1965 bis 1967 auf 3,26\% berechnen läßt (10). Wäre die Produktionszunahme ab 1967/68 wirklich bedeutend gewesen, hätten wir eine deutliche durchschnittliche jährliche Produktions- und Ertragszu- 
nahme von 1949/50 bis 1970/71 erwarten dürfen. Dem ist offensichtlich nicht so.

Würden die beiden folgenden Jahre bis 1972/73 in den Vergleich eingeschlossen, wäre infolge unregelmäßigen Monsunverlaufs überhaupt keine Ertragssteigerung festzustellen.

Trotz allen technologischen Fortschritten wird dem Witterungsablauf auch weiterhin eine bestimmende Bedeutung für die landwirtschaftlichen Erträge zukommen, da lediglich $20 \%$ der mit Getreide bebauten Flächen bewässert sind (11).

Dennoch betrug im Dürrejahr 1972/73 die Ernte 23 Millionen Tonnen mehr als bei vergleichbaren klimatischen Bedingungen 1965 bis 1967. Die Frage scheint zumindest berechtigt, ob diese Steigerung ohne die neuen Getreidesorten hätte erzielt werden können. Bei welchen Getreidearten war es bis heute möglich, die Erträge zu steigern? (siehe Tabelle 1).

Reis und Jowar scheinen von der Grünen Revolution kaum beeinflußt zu sein. Die Steigerungen bei Bajra und Mais sind relativ bescheiden, bei Weizen sensationell. Besonders nachdenklich stimmt die teilweise rückläufige Entwicklung bei den Hülsenfrüchten, de-

\section{Tabelle 1:}

Produktion und Erträge ausgewählter Nahrungsmittelgetreide, Wachstumsraten in \% pro Jahr (10)

\begin{tabular}{llrr}
\hline Getreideart & Zeitspanne & Produktion & Ertrag \\
\hline alle Nahrungs- & $1949 / 50-1964 / 65$ & 3.05 & 1.63 \\
mittelgetreide & $1949 / 50-1970 / 71$ & 3.26 & 1.99 \\
Reis & $1949 / 50-1964 / 65$ & 3.47 & 2.12 \\
& $1949 / 50-1970 / 71$ & 3.26 & 1.94 \\
Jowar (großwach- & $1949 / 50-1964 / 65$ & 2.50 & 1.49 \\
sende Hirseart) & $1949 / 50-1970 / 71$ & 1.94 & 1.18 \\
Bajra (Perlhirse) & $1949 / 50-1964 / 65$ & 2.35 & 1.23 \\
& $1949 / 50-1970 / 71$ & 3.53 & 2.23 \\
Mais & $1949 / 50-1964 / 65$ & 3.85 & 1.17 \\
& $1949 / 50-1970 / 71$ & 4.54 & 1.31 \\
Weizen & $1949 / 50-1964 / 65$ & 3.99 & 1.27 \\
& $1949 / 50-1970 / 71$ & 5.87 & 2.80 \\
Hülsenfrüchte & $1949 / 50-1964 / 65$ & 1.64 & -0.24 \\
& $1949 / 50-1970 / 71$ & 0.95 & 0.04 \\
\hline
\end{tabular}

nen als wichtigste Proteinträger für die Mehrheit der indischen Bevölkerung ein großes Gewicht bei einer qualitativen Veränderung der Ernährung zukäme.

Da der Anteil des Weizens an der gesamten Nahrungsmittelgetreideproduktion 1970/71 12,6\% betrug (zusammen mit Bajra und Mais 19,8\%) und jener von Reis sich auf $52,7 \%$ belief (zusammen mit Jowar 60\%), wird deutlich, daß ein Erfolg mit dem Anbau von Hochleistungssorten solange beschränkt bleiben muß, als ein Ertragsdurchbruch beim Reis unter indischen Bedingungen ein Traum des Nahrungsmittelgenetikers bleibt (12).

Die flächenmäßigen Anteile der neuen Getreidesorten liefern uns zusätzliche Informationen: von der gesamten Reisanbaufläche verwendeten die Bauern auf $14,7 \%$ Hochertragssorten, auch wenn ihre Resultate kaum spürbar sind. Bei Jowar betrug der Anteil $4,5 \%$, bei Bajra $1,5 \%$ und bei Mais 6,9\%. Weizen steht auch hier mit $35,7 \%$ an der Spitze.

Gesamthaft läßt sich der Anteil an Hochertragssorten auf der total verfügbaren Fläche für Nahrungsmittelgetreide in Indien auf $10,7 \%$ berechnen.

Obwohl es nicht so ist, daß die neuen Getreidesorten prinzipiell nur auf bewässertem Gebiet angebaut werden können, sollten zumindest gute Regenfälle gesichert sein. Der indische Monsun aber bleibt in seiner lokalen Verbreitung unberechenbar und läßt bis auf wenige Ausnahmen solche Gebiete nicht ausscheiden. Versuche mit Hochertragssorten sind deshalb in der Regel auf bewässerte Gebiete beschränkt. Während Düngemittel, Pestizide, spezifische Anbaukenntnisse und rechtzeitiger Zugang zu Darlehen nötig sind, um den Ertrag zu optimieren, reicht die gesicherte Bewässerung alleine dazu aus, einen großen Teil des Ertragspotentials der neuen Sorten zu nutzen.

Die meisten der leicht zugänglichen Wasserressourcen sind bereits gefaßt und eine Ausdehnung des bewässerten Landes ist zwar weiterhin möglich aber immer teurer (13).

Es gibt keinen kostenlosen, einfachen Weg für eine beschleunigte agrarische Entwicklung.

\section{Wer profitiert von den neuen Getreidesorten?}

Ohne im einzelnen an dieser Stelle die Faktoren zu nennen, die den Inder dazu bewegen, den neuen Anbaumethoden positiv oder negativ gegenüberzustehen, wollen wir in diesem Abschnitt prüfen, ob innerhalb 
jener Schicht von Bauern mit regelmäßig bewässertem Land eine Mehrheit die im Sinne einer agrarischen Modernisierung verfügbaren Innovationen übernommen hat.

In der Literatur über die Grüne Revolution wird die Frage, ob die neue Technologie in Form von Verwendung neuer Saatsorten, Düngemitteln und Pestiziden nur einer schmalen Schicht von Bauern mit großen Anbauflächen auf Kosten der zahlenmäßig dominierenden Kleinbauern zu gute kommt, sehr widersprüchlich beantwortet (14).

Wie wir festgestellt haben, ist regelmäßige Bewässerung die Grundvoraussetzung für den Anbau von neuen Getreidesorten und bestimmt die theoretische Verbreitung potentieller Adopter (15).

Zur Zeit sind systematisch geordnete und vollständige Angaben über die Verteilung des bewässerten Landes in Indien nicht verfügbar. Obwohl der Anteil an bewässertem Land von Region zu Region und von Dorf zu Dorf sehr unterschiedlich sein kann, wurde der Versuch unternommen, aufgrund verfügbarer Daten $\mathrm{zu}$ einer gesamtindischen Bilanz zu kommen (siehe Tabelle 2).

Die landwirtschaftlichen Betriebe sind in drei GröBenkategorien unterteilt: klein (I), mittel (II) und groß (III).

In der umfangreichen Literatur über Betriebsgrößen in Indien (18) finden wir keine Antwort auf die Frage, was kleine und was große Betriebe sind. Im allgemeinen aber werden jene mit weniger als 5 acres bebautem Land, ohne die Bodenqualität miteinzubeziehen, als klein bezeichnet, solche mit über 25 acres als groß. Zwölf Betriebsgrößenklassen liefern uns ein differenziertes statistisches Material.

Von unmittelbarem Interesse sind vorerst die Kolonnen 5 und 6 der Tabelle 2, die uns darüber Auskunft geben, wie das bewässerte Land über die einzelnen Größenklassen verteilt ist.

Tabelle 2: Verteilung von Landwirtschaftsbetrieben und bewässertem Land in der Indischen Union (16)

\begin{tabular}{|c|c|c|c|c|c|}
\hline \multirow{2}{*}{\multicolumn{2}{|c|}{$\begin{array}{l}\text { Größengruppen } \\
\text { (in acres) (17) }\end{array}$}} & \multirow{2}{*}{$\begin{array}{l}1 \\
\text { Anzahl landwirt- } \\
\text { schaftlicher Betriebe } \\
\text { (in 1000) }\end{array}$} & \multirow{2}{*}{$\begin{array}{l}2 \\
\text { bebautes Land } \\
\text { (in } 1000 \text { acres) }\end{array}$} & \multirow{2}{*}{$\begin{array}{l}3 \\
\text { durchschnittliche } \\
\text { Betriebsgröße } \\
\text { (in acres) }\end{array}$} & \multirow{2}{*}{$\begin{array}{l}4 \\
\text { Anzahl Betriebe } \\
\text { mit Bewässerung } \\
\text { (in 1000) }\end{array}$} \\
\hline & & & & & \\
\hline \multirow{4}{*}{\multicolumn{2}{|c|}{$\begin{array}{r}\text { I } 00.00-00.49 \\
00.50-00.99 \\
01.00-02.49 \\
02.50-04.99\end{array}$}} & 4838 & 1145 & 0.24 & 1557 \\
\hline & & 4255 & 3076 & 0.72 & 1903 \\
\hline & & 10772 & 18000 & 1.67 & 5047 \\
\hline & & 11180 & 40091 & 3.59 & 5484 \\
\hline \multirow[t]{5}{*}{ II } & 05.00-07.49 & 6158 & 37015 & 6.01 & 2957 \\
\hline & $07.50-09.99$ & 3478 & 29520 & 8.49 & 1644 \\
\hline & $10.00-14.99$ & 3881 & 45884 & 11.82 & 1787 \\
\hline & $15.00-19.99$ & 1843 & 31091 & 16.87 & 840 \\
\hline & $20.00-24.99$ & 1111 & 23891 & 21.50 & 508 \\
\hline \multirow[t]{4}{*}{ III } & $25.00-29.99$ & 663 & 17706 & 26.71 & 305 \\
\hline & $30.00-49.99$ & 1121 & 40942 & 36.52 & 482 \\
\hline & über 50.00 & 523 & 38913 & 74.40 & 204 \\
\hline & Total & 49823 & 327274 & 6.57 & 22718 \\
\hline
\end{tabular}

\footnotetext{
${ }^{1}$ Gruppendurchschnitt ${ }^{2}$ Gruppentotal
} 
Die gestellte Frage, ob die Möglichkeit zur Übernahme der neuen Technologie nur auf wenig Bauern beschränkt bleibt, kann klar beantwortet werden: falls gesicherte Bewässerung, wie wir vorerst annehmen, die notwendige und hinreichende Voraussetzung für die Übernahme von neuen Getreidesorten ist, dann können über $61 \%$ aller bewässerten Farmen, die zur Kategorie der Kleinbetriebe gehören (Kolonne 6), von den neuen Sorten profitieren. $43 \%$ der Bauern in Kategorie I (siehe Kolonne 5) verfügen über bewässertes Areal und ihr Durchschnittswert unterscheidet sich nur unwesentlich von jenen der beiden anderen Gruppen. Wenn wir Kolonne 9 betrachten ist zusätzlich anzunehmen, daß der Kleinbauer im Durchschnitt mehr Land mit neuen Getreidesorten bebaut als der Großbauer in Gruppe III.

Ohne Zweifel ist das bebaute Land ungleich verteilt. $18 \%$ der landwirtschaftlichen Betriebe der beiden untersten Größenklassen in Kategorie I (Kolonne 1) be- bauen etwas über $1 \%$ des kultivierten Landes (Kolonne 2), während knapp 5\% aller Farmen in Kategorie III 30\% des Landes kultivieren.

Das Bild verändert sich jedoch, wenn wir die Gruppenanteile des bewässerten Landes vergleichen (Kolonne 8): wenn $29 \%$ davon der Kategorie I zugerechnet werden können und die Großbauern lediglich mit 17\% vertreten sind, so kann angenommen werden, daß Kleinbauern bei der Übernahme von neuen Getreidesorten und Anbautechniken eine wichtige Rolle zu spielen haben.

Damit ist allerdings noch nicht erklärt, wieviel Bauern sich tatsächlich an der Übernahme von Hochertragssorten beteiligt haben. Leider besteht bis heute darüber nur sehr dürftiges Material. Die eigenen bis heute ausgewerteten Felduntersuchungen in Südindien und die zur Verfügung stehenden weiteren Daten aus Einzeluntersuchungen (19) haben ergeben, daß die Übernahme neuer Anbautechniken sich in allen Größen-

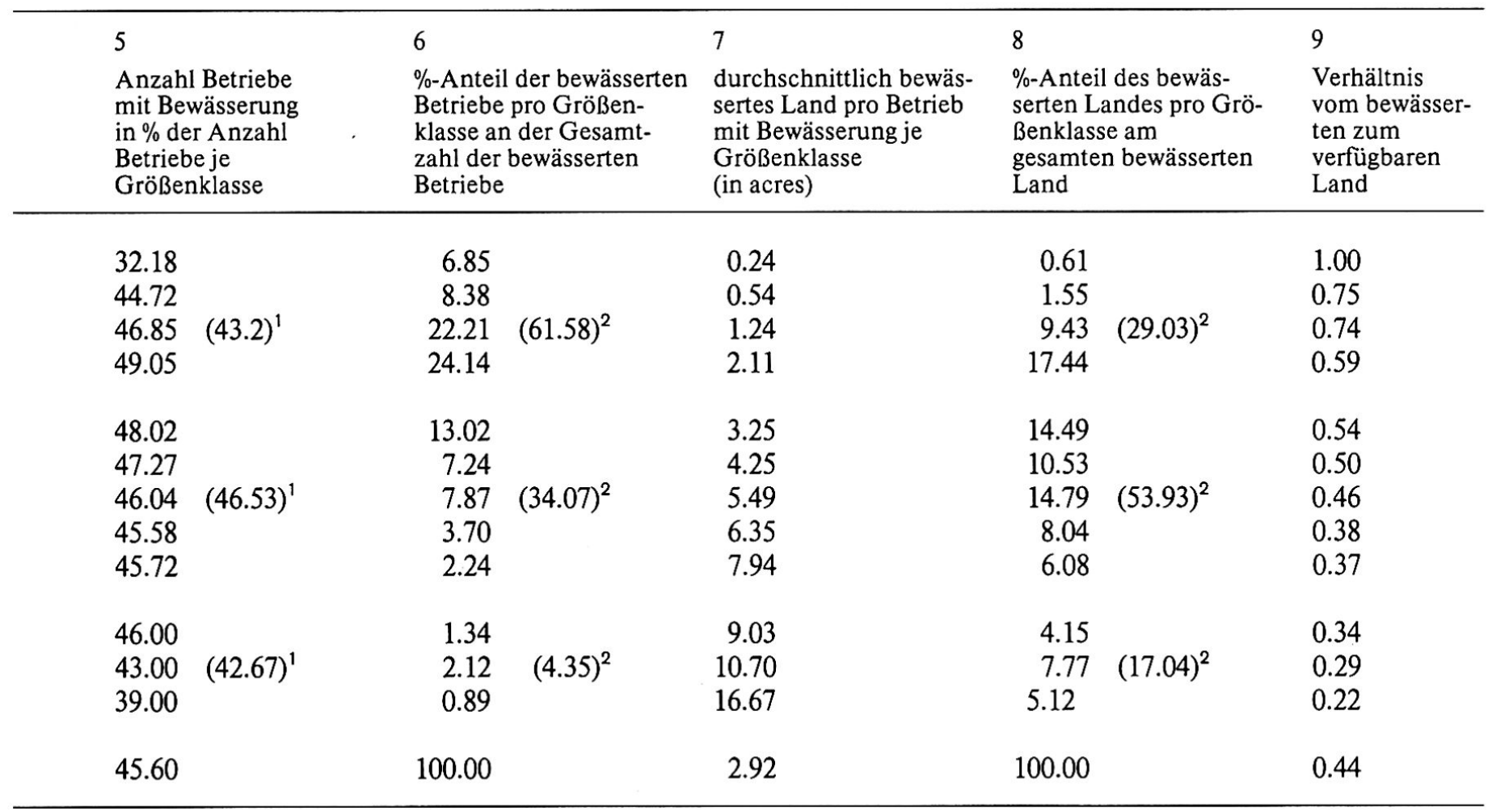


klassen relativ gleichmäßig verbreiten konnte. In den Arbeiten Mukherjee's und Lockwood's (1971) sowie in den eigenen Dorfstudien ist es gelungen, anhand von Weizenanbau im Punjab, bzw. Hirse-, Zukker- und Reiskultivationen in Karnataka - entgegen den Resultaten früherer Untersuchungen (20) nachzuweisen, daß Kleinbauern größere Neigung zeigen, agrarische Neuerungen zu übernehmen, als die Bauern der anderen beiden Größenkategorien (21).

\section{Ungewisse Zukunftsaussichten}

Zusammenfassend wollen wir festhalten, daß die Ausdehnung der Hochertragssorten in bezug auf Gesamtindien in flächen- und ertragsmäßiger Hinsicht bescheiden ist.

Schlechte Erntejahre konnten aber, nebst beträchtlichen Getreideimporten, vor allem dank einer spürbaren Ertragssteigerung in den Weizenanbaugebieten überbrückt werden.

Sollte es in den kommenden Jahren gelingen, ähnliche Erfolge beim Reis zu erzielen und durch intensive staatliche Beratung auch jene Bauern mit bewässertem Areal für den Anbau der Hochertragssorten zu überzeugen, die bisher aus verschiedenen Gründen an der Grünen Revolution nicht haben profitieren können, dann kann der heutige, allerdings qualitativ mangelhafte Ernährungsstand der indischen Bevölkerung in den kommenden 25 Jahren aufrechterhalten werden. Da es nur mit größten Anstrengungen möglich sein wird, weitere zuverlässige Wasserquellen nutzbar zu machen und über weite Gebiete vor allem Südindiens durch eine große Zahl neuer Brunnenanlagen der Grundwasserspiegel bedenklich gesunken ist, kann nicht auf eine entsprechende Ausdehnung des bewässerten Areals gesetzt werden. Zwischen verschiedenen Betriebsgrößen gibt es keine entscheidenden Unterschiede in der Übernahme neuer Getreidesorten.

Die damit verbundenen Vorteile im Sinne einer besseren Versorgung mit wirtschaftlichen Grundnahrungsmitteln und einer zunehmenden wirtschaftlichen Verflechtung (22) kann als teilweise Modernisierung des landwirtschaftlichen Entwicklungsprozesses bezeichnet werden, deren Auswirkungen sich aber zu wenig deutlich auf den gesamtwirtschaftlichen Entwicklungsprozeß übertragen haben und sich in Zukunft in ihrer Bedeutung tendenziell weiter abschwächen werden.

\section{Anmerkungen}

(1) Über die bestehenden Ungleichgewichte und Dualismen in der Dritten Welt besteht eine umfangreiche Literatur. Peter H. B., 1972, S. 64-173, gibt in seiner Dissertation einen prägnanten Überblick über die dualistische Wirtschaftsstruktur und ihre Überwindung als Grundproblem der Entwicklung. Auch Myrdal, vor allem 1972, weist in seinen Werken deutlich auf die Problematik hin.

(2) nach Statistical Outline of India, 1975. S. 11.

(3) Theoretisches Material zur Bedeutung der Landwirtschaft im wirtschaftlichen Entwicklungsprozeß liefern Johnston B. F. und Mellor J. W., 1960.

(4) Über die Schwierigkeiten, neues bebautes Land zu gewinnen, gibt die ausführliche Analyse von Farmer B. H., 1974, Auskunft.

(5) Das diesbezügliche Datenmaterial mit genauen Quellenvermerken ist in der Arbeit des Verfassers, 1974, S. 91-94, enthalten.

(6) Die Fotografie ist dem Indienbildband von Boesch H. und Imber W., 1975, S. 78, entnommen.

(7) Auf den Kranz von Faktoren, die innovationsfördernd, bzw. innovationshemmend wirken, geht der Verfasser an Hand von Einzeluntersuchungen in seiner im Entstehen begriffenen Arbeit ein. $\mathrm{Zu}$ den Begriffen Innovation, Adoption und Diffusion siehe vor allem Bronger D., 1975.

(8) Den üblichen Terminus «Grüne Revolution» haben wir bisher absichtlich nicht erwähnt, da der Verwendung von neuen Saatsorten nicht zwangsläufig eine revolutionäre agrarische Entwicklung im Sinne einer raschen Ertragssteigerung pro Flächeneinheit folgen muß. Zum Begriff und seinen Implikationen siehe Hauser J., 1972, S. 39-54. «Grüne Revolution» meint im folgenden den Anbau von HYV (High Yielding Varieties $=$ Hochertragssorten) auf eine Art und Weise, daß Produktion und Produktivität eine außergewöhnliche Steigerung aufweisen und durch verschiedene Katalysatorwirkungen und Multiplikatorprozesse (Hauser, 1972, S. 53) ein Modernisierungsproze $ß$ induziert wird.

(9) Der erste Anbau mit Hochertragssorten geht in Indien auf 1960 zurück. Mexikanische Weizensorten wurden 1963/64 ausprobiert, gefolgt von 
der tropischen Reissorte "Taichung Native 1» 1965. 1966 schließlich wurden Hochertragssorten in den Regierungsprogrammen propagiert.

(10) Wenn nicht anders vermerkt, sind diese und weitere Daten dem entstehenden Dissertations-Manuskript des Verfassers entnommen.

(11) Es dürfte sich in Wirklichkeit um einen geringeren Prozentanteil handeln, da viele der statistisch ausgewiesenen bewässerten Felder in der Trokkenzeit kein Wasser erhalten. Siehe Dakshinamurti C., 1973.

(12) $\mathrm{Zu}$ den Problemen eines erfolgreichen Reisanbaus in Indien siehe u. a. Kropp W. E., 1972, und Shenoi P. V., 1975, S. 323-325.

(13) Siehe Government of India, 1972.

(14) Siehe Staub J. und Blase M. G., 1971, und Frankel F. R., 1971.

(15) Der Anteil jener Bauern, die sich dann tatsächlich am Anbau der neuen Getreidesorten beteiligen, wird weiter hinten untersucht.

(16) Basisdaten aus Government of India, 1967 und 1968. Obwohl sich die Daten auf die beiden Landwirtschaftlichen Jahre 1959/60 und 1960/61 beziehen - es sind keine neueren Werte verfügbar geben sie einen Eindruck davon, wie zur Zeit des Beginns der Übernahme von Hochertragssorten das bewässerte Land auf die einzelnen Betriebsgrößenklassen verteilt war. Diese Bilanz hat sich bis heute nicht wesentlich verändert.

(17) 1 acre $=0.405$ ha.

(18) z. B. Krishna, 1962 oder Khusro, 1964.

(19) z. B. Muttiah C., 1971, Government of India, 1967-1969, Mukherjee P. K. und Lockwood B., 1971, Malone C. C., 1971.

(20) z. B. Ladejinsky W., 1969.

(21) Auf die zahlreichen Gründe dieses Verhaltens soll an dieser Stelle nicht näher eingegangen werden. Dazu z. B. Epstein S. T., 1973.

(22) Durch staatliche landwirtschaftliche Berater, die Aufnahme von Bankdarlehen, den Erwerb von Düngemitteln, Saatgut und Pestiziden sowie durch den Verkauf der nicht selbst benötigten landwirtschaftlichen Produktion, wird der einzelne Bauer zunehmend in regionale wirtschaftliche Aktivitäten integriert.

\section{Literaturverzeichnis}

BOESCH H. und IMBER W., Indien, Bern 1975.

BRONGER D., Der wirtschaftende Mensch in den Entwicklungsländern, Geographische Rundschau, Braunschweig No. 11, 1975, S. 449-459.

DAKSHINAMUR TI C., Water Resources of India and their Utilization in Agriculture, Water Technology Centre, Indian Agricultural Research Institute, New Delhi, 1973.

EPSTEIN S. T., South India: Yesterday, Today and Tomorrow, Mysore Villages Revisited, London 1973.

FARMER B. H., Agricultural Colonization in India since Independence, London 1974.

FRANKEL F. R., India's Green Revolution, Economic Gains and Political Costs, Princeton 1971.

GOVERNMENT OF INDIA, New Delhi

- 1967: Cabinet Secretariat, The National Sample Survey, Tables with Notes on Agricultural Holdings in Rural India, 16th Round, No. 113.

- 1967-1969: Programme Evaluation Organization, Planning Commission, Evaluating Study of the High Yielding Varieties Programme.

- 1968: Cabinet Secretariat, The National Sample Survey, Tables with Notes on Some Aspects of Land Holdings in Rural India (States and all India Estimates), 17th Round, No. 144.

- 1972: Report of the Irrigation Commission, Volume 1. HAUSER J., Die Grüne Revolution, Werden, Fortschritt und Probleme, Zürich 1972.

JOHNSTON B. F. and MELLOR J. W., The Nature of Agriculture's Contributions to Economic Development, in: Food Research Institute Studies, Stanford University, I/3, 1960, S. 335-356.

KHUSRO A. M., Returns to Scale in Indian Agriculture, Indian Journal of Agricultural Economics, Silver Jubilee Number, 1964.

KRISHNA R., The Optimum Firm and the Optimum Farm, Economic Weekly, October 6 and 13, 1962.

KROPP E. W., Betriebswirtschaftliche und sozialökonomische Aspekte des technischen Fortschritts in der Landwirtschaft der Länder Südasiens, Schriftenreihe des Forschungsinstitutes der Friedrich-Ebert-Stiftung, Band 93, Bonn-Bad Godesberg 1972, S. 235-253. 
LADEJINSKY W., The Green Revolution in Punjab: a Field Trip, Economic and Political Weekly, June 21, 1969.

MALONE C.C., Progress in Modernization of Rice, Wheat and Maize Production in Intensive Agricultural Development Districts, The Ford Foundation, New Delhi, September 1971.

MUKHERJEE P. K. and LOCKWOOD B., High Yielding Varieties Programme in India - Assessment, Paper submitted to the Seminar on the Impact of New Techniques in Agriculture, 28th International Congress of Orientalists, Canberra, January 1971.

mutTIAH C., The Green Revolution - Participation by Small versus Large Farmers, Indian Journal of Agricultural Economics, January-March, 1971.

MYRDal G., Politisches Manifest über die Armut in der Welt, Frankfurt 1972.

\section{Literaturbesprechung}

KOENZ I. U.: Guarda; 28 S. , 8 Zeichnungen, 24 Bildseiten; Paul Haupt Verlag, 1976. Fr. 9.50.

Die Reihe der Schweizer Hieimatbücher wird durch die Neuerscheinung GUARDA auf erfreuliche Weise bereichert. Der berufene Autor (Könz hat als Architekt in den 40-iger Jahren im Dorf bedeutende Restaurationen durchgeführt) äus sert sich kurz zur Landwirtschaft, die heute in den Berggebieten Europas ums Ueberleben kämpft. Anschliessend widmet er sich dem Verkehr und dem Gastgewerbe, von deren früheren Bedeutung zahlreiche Susten und Herbergen zeugen. Hauptanliegen dieses gediegenen Buches aber ist die Darstellung des Dorfes mit seinen reichgeschmückten "Engadinerhäusern" und den Sitten und Bräuchen seiner Einwohner. Vom aktuellen Kulturlandschaftswandel im Gebirge, vom Umbruch der heutigen Zeit, berichtet das kurzgehaltene Schlusskapitel. Der vorliegende, reich illustrierte Band 179 der Schweizer Heimatbücher, ist als Dokumentation einer dörflichen, alpinen Lebensgemeinschaft gerade in der Zeit tiefgreifenden Wandels einer weitgestreuten Leserschaft zu empfehlen.

Gerhard Furrer
PETER H. B., Sozialökonomische Grundprobleme der Entwicklungsländer, Zürcher Dissertation, 1972.

RAMBOUSEK w., Ausgewählte Agrarprobleme Indiens, Diplomarbeit Geographisches Institut der Universität Zürich, 1974.

RAMBOUSEK W., Indian Agriculture between Marginality and Integration - an Economic-Geographical Analysis of Four Test Villages in South India, Dissertations-Manuskript in Erarbeitung.

SHENOI P.V., Agricultural Development in India, a New Strategy in Management, Delhi 1975.

STATISTICAL OUTLINE OF INDIA, 1975, Tata Services Ltd., Bombay 1974.

STAUB J. and BLASE M. G., Genetic Technology and Agricultural Development, Science, July 9, 1971.

KELLMANN M.C.:Plant Geography; Verlag Methuen \& Co Ltd, London 1975.

Das handliche Bändchen will Geographiestudenten mit geringeren biologischen Vorkenntnissen einen konzeptionellen Rahmen zum Studium der Planzengeographie samt der dazu notwendigen Einführung in die Pflanzenbiologie vermitteln. Dabei wird das Hauptgewicht auf die fundamentalen Fragen zum Verständnis der Pflanzendekke als einen Bestandteil der Landschaft gelegt, z.B.die räumliche Verteilung der Vegetation oder einzelner ihrer Komponenten, die Methoden der Vegetationsanalyse, der Einfluss des Menschen auf die Pflanzendecke, etc. Also ein sehr verdienstvolles Unterfangen, mangelt es doch ausgesprochen an einem derartig konzentrierten, geographischen Bedürfnissen gerecht werdenden Textbuch. Leider aber vermag der Inhalt diesem hochgesteckten Ziel auf dem knapp bemessenen Raum nicht immer völlig gerecht zu werden. Dazu kommt, dass der Verfasser zu einigen zentralen Themen der modernen Pflanzengeographie, insbesondere zum Oekosystem-Konzept, in recht eigenwilliger Weise Stellung nimmt. Harold Haefner 